\title{
SEPARATION OF SOFT AND HARD PHYSICS IN DVCS
}

\author{
A. GÅRDESTIG, A.P. SZCZEPANIAK AND J.T. LONDERGAN \\ Indiana University Nuclear Theory Center, \\ 2401 Milo B. Sampson Lane, \\ Bloomington, IN 47408, USA \\ E-mail: agardest@indiana.edu
}

\begin{abstract}
A model for deeply virtual Compton scattering, based on analytical light-cone hadron wave functions is presented and studied at energies currently accessible at Jefferson Laboratory and DESY. It is shown that poles and perpendicular vector components play an important role at $Q^{2}<10 \mathrm{GeV}^{2}$. A $Q^{2}$ suppressed diagram has to be included at these low energies, but becomes negligible above $10 \mathrm{GeV}^{2}$. Future prospects and developments of this model are discussed.
\end{abstract}

\section{Introduction}

For many years, deeply inelastic scattering (DIS) has been a major source of our knowledge of parton distributions in nucleons and nuclei. This is because it can be shown that, at sufficiently high energies and momentum transfers, the amplitude for this process factorizes into a 'hard' part which can be calculated from QCD, and a 'soft' part which can be extracted from experimental data. The soft part can be proved to be related to the probability of finding a quark with a particular flavor carrying a given fraction of the nucleon's momentum.

In recent years, much interest has been focused on studying deeply virtual Compton scattering and the electroproduction of mesons, following the proof by Collins, Frankfurt and Strikman ${ }^{1}$. This proof demonstrated that, under quite general conditions, the leading amplitude for hard exclusive photo-production of mesons could also be factorized into a calculable 'hard' part, and a 'soft' part. All other amplitudes were smaller than the leading amplitude by powers of $1 / Q$. The soft part corresponds to the process by which a parton with a certain momentum fraction is removed from a nucleon, and replaced by a parton with a different momentum fraction. These parton distributions have been given a variety of names, but we will refer to them as 'generalized parton distributions' (GPD's). In the case of quark helicity conservation, there are four independent GPD's; $H(x, \zeta, t)$, 
$E(x, \zeta, t), \widetilde{H}(x, \zeta, t)$, and $\widetilde{E}(x, \zeta, t)$, where $x$ and $\zeta$ are the light-cone momentum fractions of the struck quark and real photon, while $t=\Delta^{2}$ is the momentum transfer squared. Three physically different regions could be distinguished for $x$ and $\zeta$. The domain $0<\zeta<x<1(\zeta-1<x<0)$ corresponds to the removal and replacement of a quark (antiquark) with momentum fractions $x(\zeta-x)$ and $x-\zeta(-x)$, respectively. In the remaining region $0<x<\zeta$, the photon scatters on a virtual quark-antiquark pair, extracted from the proton. With this notation, $\zeta \rightarrow x_{B}$ (Bjorken $x$ ) in the limit $Q^{2} \rightarrow \infty$ ( $\Delta$ fixed). In the limit of forward scattering (DIS), the $H$ 's reduce to the quark density and quark helicity distributions:

$$
\begin{aligned}
H(x, 0,0) & =q(x) \\
\widetilde{H}(x, 0,0) & =\Delta q(x) .
\end{aligned}
$$

The E's do not appear in DIS, they are unique to the off-forward exclusive processes and provide information not accessible through other means. The GPD's are related to the nucleon form factors by the integrals

$$
\begin{aligned}
& \int_{\zeta-1}^{1} \frac{d x}{1-\frac{\zeta}{2}} H^{q}(x, \zeta, t)=F_{1}^{q}(t), \\
& \int_{\zeta-1}^{1} \frac{d x}{1-\frac{\zeta}{2}} E^{q}(x, \zeta, t)=F_{2}^{q}(t),
\end{aligned}
$$

and similarly for $\widetilde{H}$ and $\widetilde{E}$, all independent of $\zeta$. The factor $1-\frac{\zeta}{2}$ is included to comply with the normalization of form factors used by $\mathrm{Ji}^{2}$. The GPD's might also shed some light on the nucleon spin decomposition, since $\mathrm{Ji}^{2}$ has related them to the quark spin:

$$
J_{q}=\frac{1}{2} \int_{\zeta-1}^{1} \frac{d x}{1-\frac{\zeta}{2}} x\left[H^{q}(x, \zeta, t=0)+E^{q}(x, \zeta, t=0)\right] .
$$

The quark angular momentum is decomposed into $J_{q}=S_{q}+L_{q}$, where $S_{q}$ is measured in polarized DIS experiments. If the above sum-rule is measured in DVCS, the quark spin and orbital angular momentum parts could be separated.

An alternative approach to DVCS is offered by the light-cone quark wave functions as suggested by Brodsky, Diehl, and Hwang ${ }^{3}$ (BDH). They have used this idea to calculate DVCS on an electron in QED for large $Q^{2}$.

There are ambitious efforts under way to measure DVCS (and the hard exclusive meson photo-production) at a number of facilities, DESY and JLab in particular ${ }^{4}$. At JLab energies, the competing Bremsstrahlung or Bethe-Heitler $(\mathrm{BH})$ process is larger than DVCS. However, by doing interference measurements $\left(e^{+} / e^{-}\right.$beam charge asymmetry and various spin 


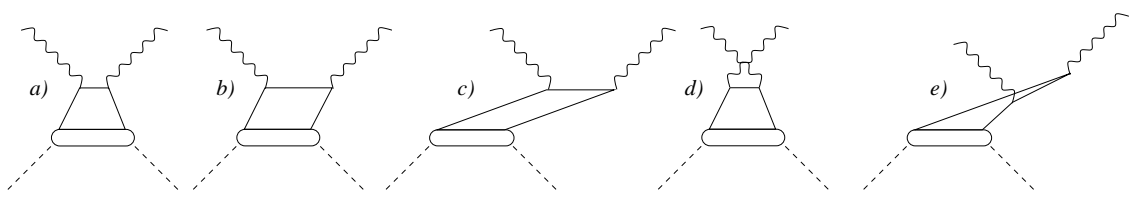

Figure 1. The five different time-ordered 'handbag' diagrams.

asymmetries) the $\mathrm{BH}$ amplitude cancels out and only a $\mathrm{BH} \times \mathrm{DVCS}$ interference remains. At DESY the energy is large enough for DVCS to become larger than $\mathrm{BH}$, though the measurements have low statistics, not allowing for differential cross sections. Data for the asymmetries are, however, at both facilities collected for relative low beam energies and momentum transfers. It is thus interesting to investigate to what extent the 'leading' amplitude is actually dominating in this kinematic region. We will here present the first few steps toward such an understanding, by developing a simple model using effective analytic quark-diquark wave functions, which allows us to study the amplitudes excited at these low energies. This model is similar to the one of BDH, but we calculate DVCS on a proton and focus especially on the features at low $Q^{2}$, keeping higher twist terms.

\section{Formalism}

We start from the Fourier transform of the $\gamma^{*} p \rightarrow \gamma p^{\prime}$ amplitude (in lightcone coordinates)

$$
T^{++}=\int d^{4} y e^{i q^{\prime} \cdot y}\left\langle p^{\prime}\left|T J^{+}(y) J^{+}(0)\right| p\right\rangle
$$

where $J^{+}(y)=\bar{\psi}(y) \gamma^{+} \gamma_{5} \frac{\tau}{\sqrt{2}} \psi(y)$ is the electromagnetic current and $p\left(p^{\prime}\right)$ and $q\left(q^{\prime}\right)$ are the four-momenta of the initial (final) hadron and photon. This expression could be expanded to give the five different one-loop diagrams (assuming light-cone gauge $q^{+}=0$ ) shown in Fig. 1. These diagrams are essentially the same as those used by BDH for DVCS on an electron. The diagrams are represented by the integrals (because of space limitations, only $a$ and $b$ are given)

$$
\begin{aligned}
T_{a}^{++} & =2\left(p^{+}\right)^{2} \int_{\zeta<x<1} \frac{d x d^{2} \mathbf{k}_{\perp}}{16 \pi^{3}} \phi^{\dagger}\left(z, \mathbf{l}_{\perp}\right) \\
& \times \frac{i}{\left[M^{2}+\left(\frac{1}{x_{B}}-1\right) Q^{2}-\frac{m^{2}+\mathbf{m}_{\perp a}^{2}}{x}-\frac{m_{R}^{2}+\mathbf{m}_{\perp}^{2}}{1-x}+i \epsilon\right]} \phi\left(x, \mathbf{k}_{\perp}\right),
\end{aligned}
$$




$$
\begin{aligned}
T_{b}^{++} & =2\left(p^{+}\right)^{2} \int_{0<x<\zeta} \frac{d x d^{2} \mathbf{k}_{\perp}}{16 \pi^{3}} \frac{\left(m_{R}^{2}-\frac{m^{2}+\mathbf{l}_{\perp}^{\prime 2}}{z^{\prime}}-\frac{M^{2}+\mathbf{l}_{\perp}^{\prime 2}}{1-z^{\prime}}\right) \varphi\left(z^{\prime}, \mathbf{l}_{\perp}^{\prime}\right)}{-\frac{\zeta\left(m^{2}+\mathbf{m}_{\perp b}^{2}\right)}{x(\zeta-x)}} \\
& \times \frac{i}{\left[M^{2}+\left(\frac{1}{x_{B}}-1\right) Q^{2}-\frac{m^{2}+\mathbf{m}_{\perp a}^{2}}{x}-\frac{m_{R}^{2}+\mathbf{m}_{\perp a}^{2}}{1-x}+i \epsilon\right]} \phi\left(x, \mathbf{k}_{\perp}\right),
\end{aligned}
$$

where $z=(x-\zeta) /(1-\zeta), z^{\prime}=(\zeta-x) /(1-x)$, and the relative momenta are defined as $\mathbf{l}_{\perp}=\mathbf{k}_{\perp}+(1-z) \Delta_{\perp}, \mathbf{l}_{\perp}^{\prime}=-\left(1-z^{\prime}\right) \mathbf{k}_{\perp}-\Delta_{\perp}, \mathbf{m}_{\perp a}=$ $\mathbf{k}_{\perp}+(1-x) \mathbf{q}_{\perp}$, and $\mathbf{m}_{\perp b}=\mathbf{k}_{\perp}+\frac{x}{\zeta} \Delta_{\perp}+\frac{\zeta-x}{\zeta} \mathbf{q}_{\perp}$. Here $x=k^{+} / p^{+}>0$ and $\zeta=q^{+} / p^{+}$are the longitudinal momentum fractions of the struck quark and the real photon, $m, m_{R}$, and $M$ the quark, remnant (diquark), and hadron masses, and $\Delta=q-q^{\prime}$. The expressions for the other diagrams have similar structures. In the limit $Q^{2} \rightarrow \infty$, the denominator of Eq. (8) is proportional to $x-\zeta+i \epsilon$, i.e., the leading twist expression of $\mathrm{Ji}^{2}$. Note that the integrals are over different parts of phase space and include scattering on a quark or quark-antiquark pair only, not scattering on an antiquark. The wave function is represented by the analytical form

$$
\phi\left(x, \mathbf{k}_{\perp}\right)=N \exp \left[-\frac{1}{\beta^{2}}\left(\frac{m^{2}}{x}+\frac{m_{R}^{2}}{1-x}+\frac{\mathbf{k}_{\perp}^{2}}{x(1-x)}\right)\right],
$$

where $\beta=0.69 \mathrm{GeV}$ is chosen such that $F\left(q^{2}\right)=\int \frac{d x d^{2} \mathbf{k}_{\perp}}{16 \pi^{3}} \phi\left(x,(1-x) \mathbf{q}_{\perp}+\right.$ $\left.\mathbf{k}_{\perp}\right) \phi\left(x, \mathbf{k}_{\perp}\right)$ agrees with the dipole form factor for $Q^{2}<1 \mathrm{GeV}^{2}$. The skew diagrams require the knowledge of the wave function $\phi\left(z^{\prime}, \mathbf{l}_{\perp}^{\prime}\right)$ for the diquark splitting into a hadron and quark (lower right-hand corner of diagrams $b, c$, and $e$ ). The form of this wave function will eventually be restricted by exclusive data, but is here arbitrarily chosen to be of the form of Eq. (9), with $m_{R}$ and $x$ replaced by $M$ and $z^{\prime}$.

\section{Results}

The $T^{++}$matrix elements have been calculated for present JLab and DESY kinematics and are shown in Fig. 2. The laboratory angle $\theta_{\gamma \gamma^{\prime}}$ between the virtual and real photons is defined for in-plane kinematics such that it is positive for $\phi=0$ and negative for $\phi=180^{\circ}$, where $\phi$ is the azimuth angle between the final electron and proton, with $\hat{\mathbf{q}}$ as the polar axis. These calculations incorporates the principal value parts of the integrals, i.e., the imaginary part of the $T^{++}$.

The forward peaking of the skew diagrams $(b, c$, and $e)$ is a consequence of the shift of the momentum $\mathbf{k}_{\perp}$ in the extra denominator. The maximum of the integral is dislocated to momenta where this denominator becomes very small. 


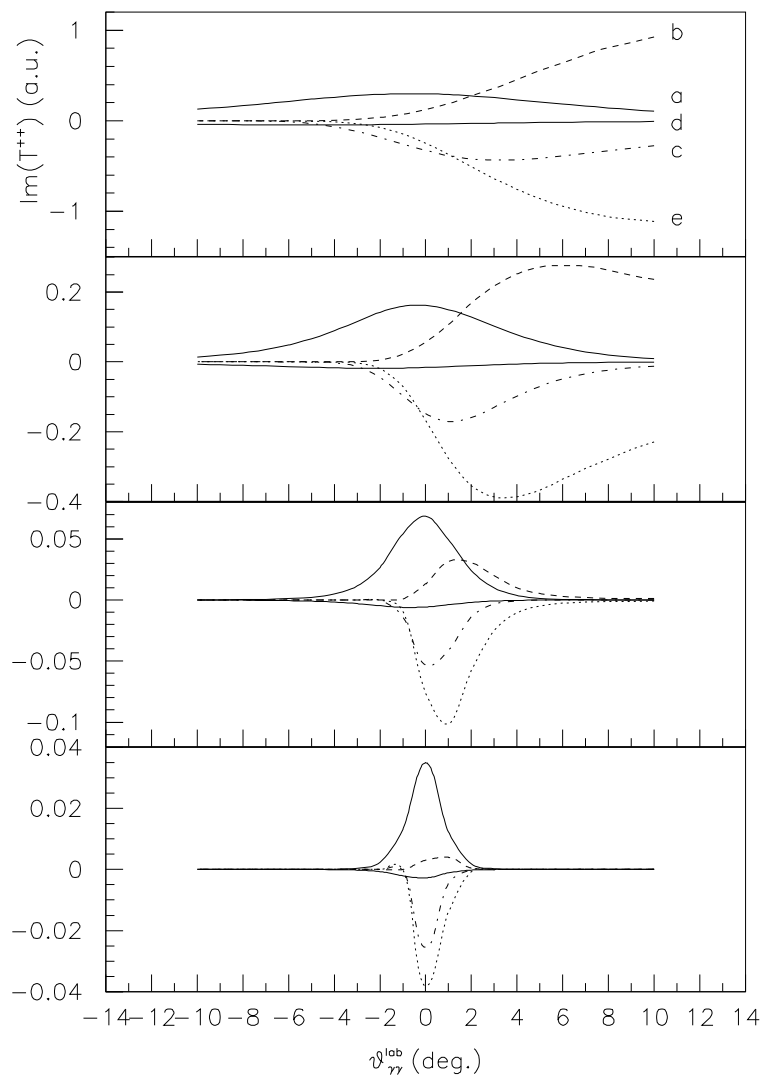

Figure 2. The $\operatorname{Im} T^{++}$matrix elements as functions of the laboratory angle between the virtual and the real photon for in-plane scattering. The labels correspond to the ones of Fig. 1 (the lower solid line always correspond to diagram $d$ ). The calculations are for $x_{B}=0.35$ and $Q^{2}=2,4,10,20 \mathrm{GeV}^{2}$ from top to bottom.

Because of its two hard propagator, diagram $b$ is expected to be suppressed in the high $Q^{2}$ limit $^{3}$, and our calculation indicates that this suppression becomes significant for $Q^{2}>10 \mathrm{GeV}^{2}$, i.e., well above present JLab energies.

While diagrams $a$ and $b$ exhibit a physical pole (for massless final photon), the other diagrams never get vanishing denominators for physical values of kinematic parameters. Thus the real part of the DVCS amplitude, which comes from the $\delta$-function part of the propagators, has contributions from these first two diagrams only. The real part calculations will not be presented here. 
This difference in pole structure also explains why diagram $d$ is smaller than diagram $a$, since $Q^{2}$ terms add up in the denominator of $d$, while they could cancel for $a$. This feature is closely related to the behavior of the two leading-twist propagators of $\mathrm{Ji}^{2} ; 1 /(x-\xi+i \epsilon)$ and $1 /(x+\xi-i \epsilon)$, where $x$ and $\xi$ are momentum fractions related to $\frac{1}{2}\left(p^{+}+p^{+}\right)$instead of $p^{+}$. In this notation there is a cancellation for $x=\xi$ (scattering on quark) or $x=-\xi$ (antiquark). These simplified propagators neglect four-vector components, e.g., $\Delta_{\perp}$, that do not give large scalars in the Bjorken limit.

\section{Conclusions and outlook}

We have introduced a model for DVCS, using simple quark wave functions and retaining all components of four-vectors throughout. The perpendicular momenta are shown to be very important for the location of the maxima of the integrals at low $Q^{2}$. Within the model we have been able to investigate the five single-loop diagrams and their relative importance for DVCS at JLab and HERA energies. The results indicate that at present JLab energies $\left(Q^{2}<4 \mathrm{GeV}^{2}\right)$, all of the diagrams (except possibly the crossed diagram $d$ ) need to be considered. In particular it is necessary to include diagram $b$, despite its two hard propagators, since the expected suppression is significant only for larger $Q^{2}$. Other higher-order diagrams might need to be considered as well. At $Q^{2}>10 \mathrm{GeV}^{2}$, the process is completely dominated by the handbag diagrams with one hard propagator.

This work will be extended to cover a wider kinematic range in $Q^{2}$ and $x_{B}$ and will include antiquark contributions to the amplitudes and the wave functions. The photo-production of mesons will also be calculated, with special consideration of the meson poles then made possible in the skew diagrams.

In order to compare with actual JLab and HERA experiments the interference with the Bremsstrahlung (Bethe-Heitler) process has to be calculated. We would then obtain cross sections and asymmetries to test against experimental results and be able to check the validity of commonly used approximations and to evaluate various wave functions that could be used.

This work was supported in part by NSF grant NSF-PHY0070368 and DOE grant DE-FG02-87ER40365.

\section{References}

1. J.C. Collins, L. Frankfurt and M. Strikman, Phys. Rev. D56, 2982 (1997).

2. X. Ji, Phys. Rev. Lett. 78, 610 (1997); Phys. Rev. D55, 7114 (1997).

3. S. J. Brodsky, M. Diehl and D. S. Hwang, Nucl. Phys. B596, 99 (2001).

4. see e.g., A. Airapetian et al., Phys. Rev. Lett. 87, 182001 (2001); S. Stepanyan et al., Phys. Rev. Lett. 87, 182002 (2001). 